\title{
Focus on pain
}

\author{
Nature Neuroscience presents a series of reviews highlighting recent progress in our understanding of the \\ neurobiology of normal and pathological pain and itch.
}

$\mathbf{P}$ ain can be defined simply as the subjective experience of harm in a part of one's body. In reality, however, there are multiple forms of pain, which involve a variety of distinct biological processes. Exposure to extreme heat, cold or pressure can be noxious, triggering nociceptive pain. Inflammatory pain, involving the release of cytokines and the infiltration of immune cells, also occurs subsequent to injury, but can be triggered independently by bacterial infections. Although pain has an important physiological role in preserving the integrity of the body, pathological pain also exists. Nerve damage, in surgery patients for instance, sometimes leads to chronic pain conditions that can last years or even decades. Unfortunately, many pathological pain conditions remain poorly understood and resist currently available treatments. Developing new therapeutic approaches to managing pain will undoubtedly depend on a better understanding of the molecular, cellular and circuit mechanisms underlying acute and chronic pain states, which is the focus of this special Nature Neuroscience issue. In this focus, we present a series of reviews by experts in the field that critically appraise recent research on the neurobiology of pain and itch.

Peripheral nociceptive neurons express a multitude of ion channels that have a key role in adaptive and maladaptive pain. These channels participate in the transduction of noxious stimuli into electrical activity in the periphery, enable the propagation of electrical signals along peripheral nerves and gate the synaptic transmission of information in the spinal cord. The voltage-gated sodium channel $\mathrm{Na}_{V} 1.7$, encoded by the SCNA9 gene, is perhaps the most striking example of a 'pain channel', as it is found exclusively in the periphery and its mutation can lead to the inability to experience pain. On page 153 of this issue, Zamponi and Waxman discuss recent advances in our understanding of the role of $\mathrm{Na}^{+}, \mathrm{K}^{+}, \mathrm{Ca}^{2+}$ and $\mathrm{Cl}^{-}$channels in the processing of noxious stimuli by primary afferent neurons. Their review also covers channel dysfunction in congenital and acquired disease states and the emerging possibilities for new analgesics based on these studies.

Like nociceptive pain, normal inflammatory pain signals that certain body parts have suffered damage and should therefore be protected from subsequent harm. However, inflammatory pain is triggered by the cross-talk between the immune and nervous systems. Interestingly, the recruitment of the immune system after tissue damage initiates a cascade of molecular and cellular processes that mediate the progressive repair of the injured tissues, thereby actively promoting healing. Many of the molecules involved in these processes are lipids and can be either proalgesic or analgesic. In a Review on page 164, Sasso and Piomelli survey the functions of endogenous lipid mediators in the peripheral gating of nociceptive signals, with a particular emphasis on the mechanisms and pathways associated with analgesic lipids, such as endocannabinoids, lipid amides, lipoxins and resolvins.

How is noxious information processed centrally? Unlike the cortex, little is known about the cellular repertoire and the wiring rules of the dorsal horn of the spinal cord. Our incomplete understanding of circuit architecture in the spinal cord and of how it connects with primary afferents may explain the lack of consensus with regard to how noxious information is processed in the CNS. The growing evidence for cross-talk between somatosensory labeled lines lends support to a theoretical framework according to which the nociceptive system operates under combinatorial encoding rules. In a Review on page 183, Prescott, Ma and De Koninck present their views on the organization of the nociceptive system and suggest that progress will require meticulous dissection of the spinal dorsal horn microcircuitry via the innovative combination of classic and modern tools.

Fundamentally, pain is a subjective experience that can vary greatly among individuals, even for identical noxious stimuli, and there is great variability in how long patients experience pain when healing from injuries. What explains these differences? What makes certain individuals more susceptible or more resilient to developing chronic pain? Early life stress, mood disorders, existing pain conditions and genetic mutations are some of the factors that can modulate the risk of triggering the transition from acute to chronic pain. In a Review on page 192, Denk, McMahon and Tracey analyze our current knowledge of the genetic, epigenetic and environmental factors that contribute to pain vulnerability and resilience and delineate the brain networks that are involved in chronic pain states.

Although itch is not pain, these two sensations share many features. Like removing our hand from a burning flame, scratching an insect bite is a response to a potentially harmful stimulus. The functions of the pruriceptive and nociceptive systems also appear to be tightly linked. Some analgesics can induce itch and some pruritogens can cause pain. Moreover, suppressing itch can cause pain and, reciprocally, inducing pain can relieve itch. As a consequence of the functional and anatomical proximity of these two systems, the study of itch has often had implications for our understanding of pain. In a Review on page 175, Bautista, Wilson and Hoon propose a synthesis of recent findings on the molecular, cellular and circuit basis of acute and chronic itch in primary sensory afferents and in the spinal cord.

These are exciting times for pain research: genomic studies are gathering momentum, studies using the newest generation of tools to manipulate and reconstruct circuits show promise, there is a growing awareness about the need to use appropriate behavioral readouts in preclinical research and to perform longitudinal studies in humans, and the results of some clinical trials look promising. Those are all good reasons to be excited about the future of the field. We would like to extend our thanks to all of the scientists who have contributed ideas, opinions, reviews and articles to this Focus issue and hope that this collection of reviews will give our readers an overview of some of the most exciting research in this field and stimulate future advances in pain research. 